\title{
Alteraciones neuropsicológicas en pacientes con aneurismas cerebrales: tratamiento quirúrgico versus tratamiento endovascular
}

C. Orozco-Giménez*; M.J. Katati; R. Vilar*; M. Meersmans*; M. Pérez-García*; J.M. Martín; P. Alcázar**; F. Guerrero***; F. Escamilla****; A. Mínguez ${ }^{* * * *}$; G. Olivares****; E. Saura; A. Jorques y V. Arjona

Departamento de Psicología*. Universidad de Granada. Servicios de Neurocirugía, Neurorradiología**, Cuidados Intensivos, ${ }^{* * *}$ y Neurología****. Hospital Universitario Virgen de las Nieves. Granada*.

Resumen

Objetivos. Describir el rendimiento neuropsicológico de pacientes con aneurismas cerebrales que han sido tratados mediante cirugía o embolización, y determinar la existencia de diferencias en función de la modalidad de tratamiento.

Material y métodos. Serie clínica compuesta por 93 pacientes voluntarios, con aneurismas cerebrales, tratados mediante cirugía $(n=56)$ o embolización $(n=37)$. A ambos grupos se les realizó una evaluación neuropsicológica retrospectiva, al menos un año después de realizar el tratamiento.

Resultados. En ambos grupos se encontraron pacientes con déficit neuropsicológicos. En el grupo de pacientes tratados quirúrgicamente el porcentaje de pacientes sin ninguna afectación neuropsicológica es del $\mathbf{3 5 . 7 \%}$, mientras que en el grupo de pacientes embolizados este porcentaje asciende al $43.2 \%$. Los análisis muestran una ejecución mejor en el grupo de tratamiento endovascular, respecto al quirúrgico, sólo en memoria visual y en el recuerdo con claves de la memoria verbal.

Conclusiones. Años después del tratamiento, un importante porcentaje de pacientes presenta alteraciones neuropsicológicas. El tratamiento endovascular se asocia con un mejor rendimiento en memoria visual y en el recuerdo con claves de la memoria verbal, aunque explica un porcentaje muy escaso de la varianza. Por lo tanto, en la explicación del deterioro neuropsicológico parece más importante el propio efecto de la hemorragia que la modalidad de intervención.

PALABRAS CLAVE: Aneurisma cerebral. Embolización. Cirugía. Cognición. Neuropsicología.

Neuropsychological impairment in patients with intracranial aneurysms: surgical versus endovascular treatment

Recibido: 9-02-04. Aceptado: 22-06-04
Summary

Objectives. To describe the neuropsychological status of patients with intracranial aneurysms and to compare the cognitive status of patients with intracranial aneurysm treated by surgical or endovascular mehtods.

Material and methods. Ninety-three cases with intracranial aneurysms treated with surgery $(n=56)$ or embolization $(n=37)$ were included. A neuropsychological assessment was applied to both groups retrospectively, at least one year after treatment.

Results. Neuropsychological impairment was found in both groups. $35.7 \%$ of the patients treated with surgery and $43.2 \%$, of those treated with embolization did not show any cognitive impairment. Visual Memory and Cued Recall of verbal information are better in patients treated by embolization.

Conclusions. Our results show that a large proportion of patients with intracranial aneurysms have cognitive impairment after treatment. Endovascular management may cause less impairment in visual and verbal memory. However, bleeding may be the most important factor to explain these cognitive impairments.

KEY WORDS: Irntracranial aneurysms. Endovascular embolization. Surgery. Cognition. Neuropsychology.

\section{Introducción}

La mortalidad entre los pacientes con hemorragia subaracnoidea espontánea, ingresados en un servicio de Neurocirugía, puede alcanzar hasta un $26 \%{ }^{16}$. Hasta hace unos años el tratamiento quirúrgico ha sido la principal estrategia para prevenir el resangrado de los aneurismas cerebrales. Los déficits cognitivos a largo plazo, asociados a la hemorragia subaracnoidea y a su tratamiento quirúrgico, han sido ampliamente descritos en la literatura. De este modo, se ha mostrado la existencia de déficits neuropsicológicos en estos pacientes, que afectan principalmente a las funciones cognitivas de memoria, atención, percep- 
ción, praxia visoespacial y función ejecutiva $a^{1,2,11,12,19}$.

Existe menos consenso respecto a qué factores clínicos y quirúrgicos podrían explicar estos déficits. Los datos publicados no son consistentes. En numerosas publicaciones la localización del aneurisma no se relaciona con el estado neuropsicológico ${ }^{5,6,20,28}$, mientras que otros trabajos sí relacionan dicha localización del aneurisma con el déficit neuropsicológico. Así, se han asociado los aneurismas situados en la circulación posterior con un mejor pronóstico para la memoria verbal y visual $^{18}$. Del mismo modo, la ruptura de los aneurismas de la arteria comunicante anterior, así como la oclusión temporal de vasos realizada durante su intervención quirúrgica, se relaciona con mayores déficit en memoria y función ejecutiva ${ }^{29}$. Respecto a otras variables clínicas, los datos siguen siendo contradictorios. En algunos estudios, el estado clínico al ingreso (WFNS o Hunt \& Hess) o la gravedad de la hemorragia (grado Fisher) no presentan relación con el funcionamiento neuropsicológico ${ }^{23,27}$, mientras que en otras publicaciones estos factores sí se relacionan con un peor pronóstico en algunas variables neuropsicológicas ${ }^{5,12,18}$. Por último, también se han considerado factores quirúrgicos y periquirúrgicos como el resangrado, el vasoespasmo, la hidrocefalia o el infarto cerebral, no encontrándose relación consistente entre estos y algunas variables neuropsicológicas ${ }^{1,18,23}$.

Hasta el momento, no se ha podido revelar el grado de contribución de la cirugía a los déficit cognitivos de estos pacientes, por ausencia hasta hace poco de adecuados grupos de control. En 1991 Gugliemi $^{8}$ introduce el tratamiento endovascular, que pronto se convierte en una alternativa terapéutica eficaz en el tratamiento de los aneurismas cerebrales. Las diferencias entre ambos tipos de tratamiento, en términos de morbimortalidad, se han discutido ampliamente en la literatura ${ }^{21}$. No obstante, las posibles diferencias en las consecuencias neuropsicológicas han comenzado a estudiarse recientemente. Por tanto, hay pocos estudios que hayan explorado las funciones cognitivas y psicológicas de pacientes tratados de aneurismas cerebrales, en comparación con los tratados con embolización. Hadjivassiliou y colaboradores, en $2001^{9}$, llevaron a cabo un estudio comparativo con 40 pacientes con aneurismas cerebrales rotos sometidos a tratamiento endovascular, y 40 sometidos a tratamiento quirúrgico. Ambos grupos eran homogéneos en el WFNS, en el momento de la admisión, edad y localización del aneurisma. Los autores encontraron que el grupo de tratamiento endovascular presentaba un mejor funcionamiento neuropsicológico en comparación con el grupo de tratamiento quirúrgico. Esta tendencia resultó estadísticamente significativa en memoria visual, función ejecutiva, y en el subtest vocabulario de la Escala de Inteligencia para Adultos Weshler - Revisada ${ }^{30}$. En este mismo trabajo, los autores encontraron mayor presencia de encefalomalacia en las imágenes por resonancia magnética entre los pacientes tratados quirúrgicamente en comparación a los pacientes embolizados. Chan y colaboradores también en el año $2001^{3}$ han estudiado las diferencias en funcionamiento cognitivo en pacientes tratados de aneurisma de la arteria comunicante anterior con embolización o cirugía. En una pequeña serie, compuesta por 9 pacientes de cada grupo, los autores encontraron que una tercera parte de los pacientes tratados quirúrgicamente mostraban deterioro neuropsicológico, mientras que dicho deterioro no aparecía en el grupo tratado con embolización. En un reciente trabajo de Fontanella y colaboradores ${ }^{7}$ no han encontrado diferencias significativas entre los pacientes con aneurismas cerebrales que reciben tratamiento endovascular y quirúrgico. No obstante, los autores muestran que el grupo de pacientes tratados con cirugía presenta una ejecución peor que los controles intactos, en lenguaje y en las funciones del lóbulo frontal, mientras que el grupo tratado con embolización no se diferenciaba de los controles sanos.

Los objetivos de este estudio son, en primer lugar, describir el funcionamiento cognitivo de los pacientes con aneurismas cerebrales tratados quirúrgica y endovascularmente. En segundo lugar, queremos determinar si existen diferencias en el funcionamiento cognitivo entre ambos grupos.

\section{Material y métodos}

\section{Pacientes}

Entre 1995 y Febrero de 2002 se trataron 204 pacientes con aneurismas cerebrales en el Servicio de Neurocirugía del Hospital Universitario Virgen de las Nieves. Ciento cuatro pacientes aceptaron participar en este estudio retrospectivo, y finalmente se incluyen para este estudio noventa y tres $(45,59 \%)$, ya que dos pacientes presentaban otras alteraciones que tenían asociados déficits neuropsicológicos (Parkinson y traumatismo craneoencefálico), cuatro presentaban un episodio depresivo mayor, uno no hablaba correctamente la lengua española, uno presentaba un síndrome afásico que impidió la aplicación de toda la batería y tres mostraron falta de colaboración durante la evaluación. Los pacientes recibieron tratamiento quirúrgico o endovascular según su adecuación. Cincuenta y seis de los pacientes fueron tratados quirúrgicamente $(60,22 \%)$, mientras que los restantes treinta y siete fueron tratados con embolización $(38,78 \%)$. Las características demográficas, clínicas y quirúrgicas de los pacientes se muestran en las tablas 1 y 2. 
Tabla 1

Diferencias en la distribución de las variables demográficas y clínicas entre los grupos de tratamiento quirúrgico y endovascular

Variable

\section{Cirugía}

$n(\%)$

Sexo

\section{Mujer}

Hombre

$34(60,7)$

$22(39,3)$

Dominancia Corporal

Diestro

Zurdo

Localización del aneurisma

Comunicante anterior

Cerebral media

Comunicante posterior

Bifurcación carótida

Otras

Hunt \& Hess

0 (intacto)

1-3 (moderado)

4-5 (severo)
$52(92,9)$

$4(7,1)$

$30(53.6)$

11 (19.6)

$4(7.1)$

$4(7.1)$

$7(12.5)$
$5(8.9)$

$42(75)$

$9(16.1)$
Embolización

$n(\%)$

$19(51.4)$

18 (48.6)

$37(100)$

$0(0)$

$10(27)$

$5(13.5)$

$10(27)$

$2(5.4)$

$10(27)$
$<0.150$

$<0.050$

ns

$<0.150$

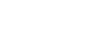

Fisher

I

II

III

$1 \mathrm{~V}$
$4(10.8)$

$27(73)$

$6(16.2)$ ns

$6(16.2)$

$12(32.4)$

$4(10.8)$

$15(40.5)$

Miscelancas / Complicaciones

Drenaje ventricular

DVh

Resangrado antes tratamiento

Hidrocefalia

Vasoespasmo

Infarto

Rotura intraoperatoria
$11(19.6)$

$13(23.2)$

$17(30.49)$

$15(26.8)$
$5(13.5)$

7 (18.9)

1 (2.7)

7 (18.9)

13 (35.1)

8 (21.6)

$0(0)$
$<0.150$

ns

ns

ns

ns

ns

ns

ns

$4(10.8)$

ns 
Tabla 2

Diferencias en las medias de las variables clínicas y demográficas de los grupos de tratamiento quirúrgico y endovascular

\begin{tabular}{llllll} 
Variable & Media & DT & Min - Max & $P$ \\
\hline Edad en la evaluación & & & & & \\
$\quad$ Cirugía & 54 & 11.10 & 28 & 78 & ns \\
$\quad$ Embolización & 54 & 13.64 & 26 & 78 & \\
\hline
\end{tabular}

Edad en el tratamiento

$\begin{array}{llllll}\text { Cirugía } & 49.55 & 11.55 & 24 & 75 & \mathrm{~ns} \\ \text { Embolización } & 50.43 & 13.71 & 20 & 75 & \end{array}$

Tiempo de evolución (años)

$\begin{array}{llllll}\text { Cirugia } & 3.95 & 2.23 & 1 & 7.92 & \text { ns } \\ \text { Embolización } & 3.69 & 1.44 & 1.7 & 6.17 & \end{array}$

\begin{tabular}{llllll} 
Años de escolaridad & & & & \\
Cirugía & 6.75 & 4.49 & 0 & 16 & ns \\
Embolización & 8 & 5.24 & 0 & 18 & \\
\hline
\end{tabular}

DT = Desviación típica; Min - Max = Mínimo y Máximo; ns = no significativo

\section{Material}

La NEUROPSI es una batería neuropsicológica breve que consta de diversas tareas y que ha sido validada y baremada ${ }^{24,25}$. Permite establecer el funcionamiento neuropsicológico en todos los pacientes, incluyendo aquéllos muy deteriorados neuropsicológicamente y aquellos pacientes analfabetos o con un nivel nulo de escolaridad según los autores ${ }^{24,25}$. Una parte importante de nuestros pacientes no fueron escolarizados, por lo que eliminamos de la batería las tareas que requerían alfabetización y conocimientos académicos. En el estudio tampoco mostraremos los resultados de aquellas tareas que presentaron efecto techo al no discriminar entre los pacientes (orientación, denominación, y cambio de mano derecha e izquierda). Se han seleccionado las siguientes tareas:

\section{- Funciones motoras:}

1. Movimiento alternos de las manos. Consiste en realizar movimientos alternativos con las manos (mano derecha abierta y mano izquierda cerrada, y viceversa). El examinador realiza primero los movimientos y después se le pide al paciente que los realice.

2. Reacciones opuestas. El ejercicio consiste en que, si el examinador muestra un dedo, el paciente debe mostrar el puño, mientras que si el examinador muestra el puño el paciente debe mostrar el dedo.

\section{- Atención y concentración:}

3. Dígitos hacia atrás. Repetición a la inversa de series de dos a seis dígitos.

4. Detección visual. Test de cancelación con 16 tipos de figuras distintas, cada una de las cuales se repite 16 veces. El paciente debe tachar una determinada figura que se le ha mostrado previamente durante tres segundos. El test se suspende tras un minuto.

5. Resta 3. El paciente ha de restar desde 20 de tres en tres hasta 5 .

\section{- Lenguaje:}

6. Comprensión. Esta es una prueba similiar al Token Test ${ }^{4}$. En una hoja se presentan cuatro figuras (círculos y cuadrados grandes y pequeños) que el paciente debe tocar, según se le indica en seis instrucciones diferentes.

7. Fluidez verbal (animales). En un minuto el paciente ha de nombrar cuantos animales pueda recordar.

\section{- Codificación:}

8. Memoria verbal (codificación). Se presentan, en tres ensayos, seis nombres comunes agrupados en tres categorías semánticas (frutas, animales y partes del cuerpo). Después de cada ensayo, el paciente repite las palabras que puede recordar. La variable de resultado es la media aritmética del total de palabras recordadas en los tres ensayos.

9. Copia de una figura semicompleja. Se pide al paciente que 
copie una figura similar a la Figura Compleja de Rey ${ }^{26}$ pero más sencilla.

\section{- Recuerdo:}

10. Recuerdo libre de la figura semicompleja. Recuerdo espontáneo de la figura copiada en la tarea de copia de una figura semicompleja.

11. Recuerdo libre de la información verbal. Recuerdo espontáneo de las seis palabras presentadas en los ensayos de codificación verbal.

12. Recuerdo con claves de la información verbal. Se pide al paciente que recuerde qué animales, frutas y partes del cuerpo se le dijeron.

13. Reconocimiento de la información verbal. El examinador lee 14 palabras y el paciente debe elegir cuáles se le presentaron anteriormente.

\section{Funciones conceptuales:}

14. Semejanzas. Se presentan tres pares de palabras y se le pregunta al paciente cuál es la semejanza.

\section{Procedimiento}

\section{Procedimiento quirúrgico y endovascular}

En la unidad de cirugía vascular de nuestro hospital, inicialmente todos los pacientes con aneurismas cerebrales son valorados para tratamiento endovascular. El tratamiento quirúrgico se realiza en los casos en los que la embolización no puede llevarse a cabo por ser el aneurisma de cuello ancho o por la presencia de algún vaso eferente del propio saco aneurismático.

\section{Evaluación neuropsicológica}

Tras haber transcurrido un mínimo de un año tras el tratamiento se citó a los paciente para realizar una evaluación neuropsicológica. Los 93 pacientes fueron evaluados en una única sesión de aproximadamente una hora por neuropsicólogos entrenados (C.O. \& R.V.).

Las puntuaciones directas de cada paciente se convirtieron a puntuaciones estandarizadas llamadas puntuaciones $\mathrm{T}($ Media $=$ 50, Desviación Típica $=10$ ), que permiten conocer la ejecución del paciente corrigiendo el efecto de la edad y la escolaridad.

\section{Análisis estadísticos}

En primer lugar, con el objetivo de establecer si ambos grupos son homogéneos en los diferentes factores demográficos, clínicos y quirúrgicos, se realizan análisis de contingencia para variables nominales y $\mathrm{T}$ de Student para variables continuas. Se considera que existe una tendencia a la significación si $p<0.150$. En segundo lugar, y para determinar la presencia de diferencias en el funcionamiento neuropsicológico entre ambos grupos, se realizan análisis $\mathrm{T}$ de Student para comparar medias de muestras independientes. Se realiza el test de Levene de homogeneidad de las varianzas para determinar qué estadístico T de Student emplear.
2006; 17: 34-45

En tercer lugar, con el objetivo de considerar el efecto de las variables demográficas, clínicas o quirúrgicas en las que los grupos no fueran homogéneos, se aplica un análisis de regresión lineal. Se consideran como variables dependientes o predichas las variables de la batería NEUROPSI descritas en el epígrafe "Material". Se consideran variables independientes o predictoras, la modalidad de tratamiento (endovascular o quirúrgica) y aquellas variables demográficas y clínicas en las que exista una tendencia a la significación $(\mathrm{p}<0.150)$. Para estudiar las variables predictoras categóricas, se crean variables auxiliares o variables "dummy", que permiten estudiar el efecto de los diferentes niveles de las variables categóricas respecto al nivel de control (p.e. ausencia de daño) o respecto al nivel más frecuente.

\section{Resultados}

En primer lugar, con el objetivo de determinar si los grupos eran homogéneos en características clínicas y demográficas se realizaron 13 análisis de contingencia y $4 \mathrm{~T}$ de Student (ver tablas 1 y 2). Dichos análisis no resultaron estadísticamente significativos, excepto en la distribución de la variable localización del aneurisma $\left(\chi^{2}=12.664, \mathrm{p}=0.013\right)$. No obstante, las variables dominancia manual y la extensión de la hemorragia subaracnoidea indicada por el grado Fisher, mostraron una tendencia a la significación $(\mathrm{p}<0.150)$. En las tablas 1 y 2 se resumen las características principales de cada uno de los dos grupos de tratamiento y si existen diferencias estadísticamente significativas en cada una de las variables entre ambos grupos.

En segundo lugar, y con el objetivo de describir el funcionamiento cognitivo de ambos grupos de pacientes, se determinó el número de pacientes que presentan deteriorada la ejecución en alguna de las tareas de la prueba. En la tabla 3 se muestra el número de pacientes que presentan puntuaciones $2 \mathrm{SD}$ inferiores a la media (puntuación $\mathrm{T}<30$ ) para cada variables estudiada. Como se observa, la función más frecuentemente afecta en los pacientes quirúrgicos es la comprensión (21.4\%), y la menos afectada la variable dígitos hacia atrás (3.6\%). Dentro del grupo de tratamiento quirúrgico, el $35.7 \%$ de los pacientes no presentaron deterioro en ninguna de las variables, es decir, se encuentran neuropsicológicamente intactos. Por otro lado, en el grupo de pacientes tratados endovascularmente, la variable más afectada es memoria verbal (codificación) afectando al 18.9\% de los pacientes. Las variables que presentan una menor incidencia del deterioro neuropsicológico son dígitos hacia atrás, resta 3 , fluidez verbal, recuerdo libre de información verbal, recuerdo con claves de información verbal y semejanzas $(2.7 \%)$. El porcentaje de pacientes con todas las variables intactas es de $43.2 \%$.

Para determinar la existencia de diferencias en el funcionamiento cognitivo, debidas a la modalidad de tratamiento recibido (endovascular versus quirúrgico), se realizaron diversos análisis. Primero de ello, se llevaron a cabo catorce T de Student con las catorce variables neuropsicológicas como variables dependientes, y el tipo de tratamiento como variable independiente. Los 
Tabla 3

Frecuencia y porcentajes de deterioro neuropsicológico en el grupo de tratamiento quirúrgico y endovascular

\begin{tabular}{lcc}
\hline Variable & Cirugía & Embolización \\
& $n(\%)$ & $n(\%)$ \\
\hline Movimiento alternos de las manos & & $5(13.5)$ \\
Reacciones opuestas & $4(7.1)$ & $2(5.4)$ \\
Dígitos hacia atrás & $3(5.4)$ & $1(2.7)$ \\
Detección visual & $2(3.6)$ & $5(13.5)$ \\
Resta 3 & $8(14.3)$ & $1(2.7)$ \\
Comprensión & $6(10.7)$ & $6(16.2)$ \\
Fluidez verbal & $12(21.4)$ & $1(2.7)$ \\
Memoria verbal (codificación) & $4(7.1)$ & $7(18.9)$ \\
Copia de una figura semicompleja & $8(14.3)$ & $3(8.1)$ \\
Recuerdo libre de la figura semicompleja & $13(23.2)$ & $1(2.7)$ \\
Recuerdo libre de la información verbal & $15(26.8)$ & $1(2.7)$ \\
Recuerdo con claves de la información verbal & $9(16.1)$ & $2(5.4)$ \\
Reconocimiento de la información verbal & $10(17.9)$ & $4(10.8)$ \\
Semejanzas & $3(5.4)$ & $1(2.7)$ \\
\hline
\end{tabular}

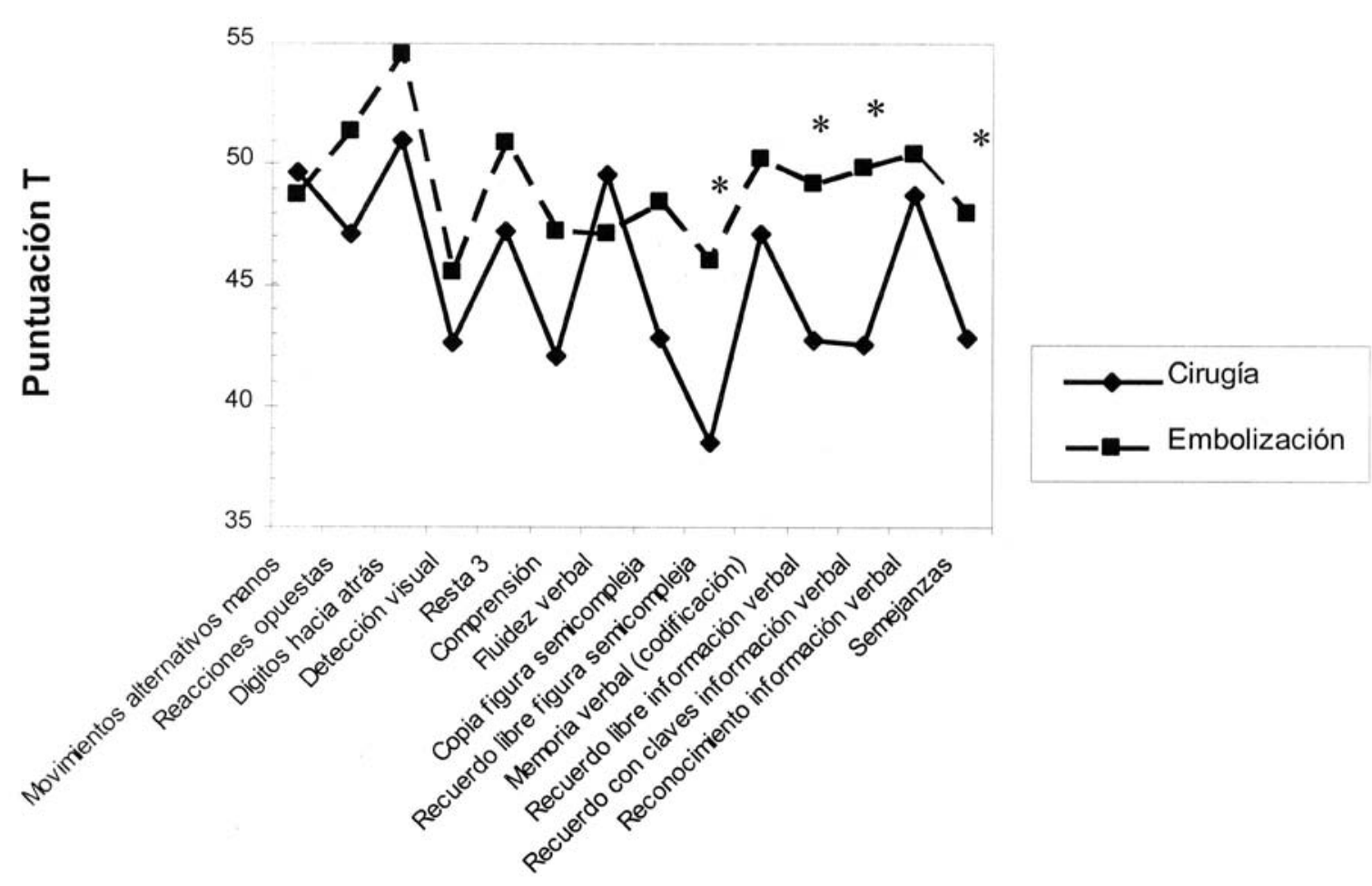

* : Diferencias esadísticamente significativas en la pureba $t$ de Student $(\mathrm{p}<0.05)$

Figura 1. Perfil de puntuaciones medias de los grupos de tratamiento quirúrgico y endovascular en cada tarea de la batería neuropsicológica NEUROPSI. 
Tabla 4

Relación entre las variables dominancia manual, grado Fisher, localización del aneurisma y modalidad de tratamiento con el rendimiento neuropsicológico

\begin{tabular}{|c|c|c|c|c|c|c|c|}
\hline Variable Dependiente & $\begin{array}{c}\mathrm{R}^{2} \text { Ajustad } \\
\mathrm{a}\end{array}$ & $\mathrm{F}$ & $p$ & Predictores & $\beta$ & $\mathrm{T}$ & $\mathrm{p}$ \\
\hline Movimiento alternos de las manos & 7.4 & 3.591 & 0.032 & $\begin{array}{l}\text { Fisher Grado II } \\
\text { Tratamiento }\end{array}$ & $\begin{array}{l}8.483 \\
1.665\end{array}$ & $\begin{array}{l}2.662 \\
0.577\end{array}$ & $\begin{array}{c}0.009 \\
\mathrm{~ns}\end{array}$ \\
\hline Digitos hacia atrás & 7.1 & 3.426 & 0.037 & $\begin{array}{l}\text { CoP } \\
\text { Tratamiento }\end{array}$ & $\begin{array}{r}6.434 \\
-2.287\end{array}$ & $\begin{array}{l}2.056 \\
1.000\end{array}$ & $\begin{array}{c}0.043 \\
\text { ns }\end{array}$ \\
\hline Detección visual & 8.7 & 4.310 & 0.016 & $\begin{array}{l}\text { Fisher Grado IV } \\
\text { Tratamiento }\end{array}$ & $\begin{array}{l}-8.111 \\
-4.084\end{array}$ & $\begin{array}{l}2.730 \\
1.443\end{array}$ & $\begin{array}{c}0.007 \\
\text { ns }\end{array}$ \\
\hline Resta 3 & 6.3 & 4.111 & 0.020 & $\begin{array}{l}\text { Fisher Grado IV } \\
\text { Tratamiento }\end{array}$ & $\begin{array}{l}-5.921 \\
-4.457\end{array}$ & $\begin{array}{l}2.403 \\
1.894\end{array}$ & $\begin{array}{c}0.018 \\
n s\end{array}$ \\
\hline Copia de una figura semicompleja & 12.9 & 4.400 & 0.003 & $\begin{array}{l}\text { Dominancia } \\
\text { Fisher Grado II } \\
\text { BCa } \\
\text { Tratamiento }\end{array}$ & $\begin{array}{r}13.181 \\
5.744 \\
13.953 \\
-5.351\end{array}$ & $\begin{array}{l}1.1722 \\
1.643 \\
2.232 \\
1.691\end{array}$ & $\begin{array}{c}\mathrm{ns} \\
\mathrm{ns} \\
0.028 \\
\mathrm{~ns}\end{array}$ \\
\hline $\begin{array}{l}\text { Recuerdo libre de la figura } \\
\text { semicompleja }\end{array}$ & 19.7 & 8.511 & $>0.001$ & $\begin{array}{l}\text { Dominancia } \\
\text { BCa } \\
\text { Tratamiento }\end{array}$ & $\begin{array}{l}21.530 \\
19.105 \\
-6.297\end{array}$ & $\begin{array}{l}2.886 \\
3.146 \\
2.038\end{array}$ & $\begin{array}{l}0.005 \\
0.002 \\
0.044\end{array}$ \\
\hline $\begin{array}{l}\text { Recuerdo libre de la información } \\
\text { verbal }\end{array}$ & 10.9 & 4.767 & 0.004 & $\begin{array}{l}\text { Dominancia } \\
\text { CoP } \\
\text { Tratamiento }\end{array}$ & $\begin{array}{r}13.609 \\
5.834 \\
-4.276\end{array}$ & $\begin{array}{l}2.203 \\
1.626 \\
1.1613\end{array}$ & $\begin{array}{c}0.030 \\
\mathrm{~ns} \\
\mathrm{~ns}\end{array}$ \\
\hline $\begin{array}{l}\text { Recuerdo con claves de la } \\
\text { información verbal }\end{array}$ & 13.6 & 5.824 & 0.001 & $\begin{array}{l}\text { Dominancia } \\
\text { BCa } \\
\text { Tratamiento }\end{array}$ & $\begin{array}{r}16.526 \\
-10.952 \\
-5.913\end{array}$ & $\begin{array}{l}2.527 \\
2.057 \\
2.183\end{array}$ & $\begin{array}{l}0.013 \\
0.043 \\
0.032\end{array}$ \\
\hline Semejanzas & 9.1 & 4.078 & 0.009 & $\begin{array}{l}\mathrm{BCa} \\
\mathrm{CoP} \\
\text { Tratamiento }\end{array}$ & $\begin{array}{r}11.088 \\
4.786 \\
-4.406\end{array}$ & $\begin{array}{l}2.400 \\
1.452 \\
1.839\end{array}$ & $\begin{array}{c}0.018 \\
\mathrm{~ns} \\
\mathrm{~ns}\end{array}$ \\
\hline
\end{tabular}

CoP: Arteria comunicante anterior; BCa: bifurcación de la arteria carótida.

resultados se muestran en la figura 1. El grupo de tratamiento endovascular presentó una ejecución estadísticamente mayor en las variables copia de la figura semicompleja $\left(\mathrm{t}_{90.592}=-2.211 ; p=\right.$ $0.030)$, recuerdo libre de la figura semicompleja $\left(\mathrm{t}_{90.384}=-2.490 ; p\right.$ $=0.015)$, recuerdo libre de la información verbal $\left(\mathrm{t}_{91}=-2.465 ; p=\right.$ $0.016)$, recuerdo con claves de la información verbal $\left(\mathrm{t}_{91}=-2.620\right.$; $p=0.010)$ y semejanzas $\left(\mathrm{t}_{90.592}=-2.338 ; p=0.022\right)$ (figura 1$)$.

No obstante, hemos de considerar que la distribución de las variables localización del aneurisma, la dominancia corporal y grado Fisher, no era la misma en ambos grupos. Dado que estas variables podrían estar explicando el funcionamiento neuropsicológico de los pacientes, se decidió aplicar un análisis de regresión lineal que mostrara si cada una de estas variables, junto con el tipo de tratamiento, afectaban al funcionamiento neuropsicológico. Se aplicaron entonces, 14 análisis de regresión lineal, considerándose como variables dependientes o predichas las variables de la batería NEUROPSI descritas. Se consideraron variables independientes o predictoras, la modalidad de tratamiento (endovascular o quirúrgica), la localización de aneurisma, la dominancia corporal (diestras o zurda) y el grado Fisher. Se 
realizaron cuatro bloques de variables predictoras. El primer bloque incluyó la dominancia manual. El segundo bloque incluyó 3 variables auxiliares o "dummy" que indicarán la presencia o ausencia de las categorías del grado Fisher II, III y IV.

Las variables "dummy" permitieron estudiar el efecto de los grados Fisher incluidos, en comparación con el grado I o control. El tercer bloque incluyó las variables auxiliares o "dummy" que indicaban la presencia o ausencia de las categorías localización del aneurisma de la arteria cerebral media, en la arteria comunicante posterior, en la bifurcación de la carótida o en otras localizaciones (aneurismas de las arterias oftálmica, pericallosa, cerebelosa posteroinferior, basilar, carotídeo intracavernoso y cerebral posterior). De este modo se determinará el efecto de la localización del aneurisma en comparación con la categoría más frecuente (aneurismas de la arteria comunicante anterior). En los tres bloques se empleó como método de selección de variables pasos sucesivos, y como criterio de entrada una probabilidad de F inferior a 0.05 , y de salida superior a 0.10 . Por ultimo, en el cuarto bloque se incluyó la modalidad de tratamiento (endovascular o quirúrgica) empleándose el método "introducir para seleccionar las variables". Los resultados de las ecuaciones estadísticamente significativas se muestran en la tabla 4. Las ecuaciones para las variables reacciones opuestas, comprensión, fluidez verbal, memoria verbal (codificación) y reconocimiento de la información verbal, no resultaron significativas con el modelo seleccionado. Para las demás variables, las ecuaciones resultaron estadísticamente significativas, aunque con un nivel de ajuste muy bajo, ya que el porcentaje de varianza explicado oscila entre $6.3 \%$ y $19.7 \%$. A continuación, describimos el efecto que cada una de las variables predictoras tiene sobre los dos dominios de la calidad de vida que son predichos por el modelo empleado.

\section{Efecto de la dominancia}

La dominancia manual predice significativamente las variables recuerdo libre de la figura semicompleja, recuerdo libre de la información verbal y recuerdo con claves de la información verbal. En todos los casos, y manteniéndose constante el resto de variables, los pacientes diestros presentan una mejor puntuación frente a los pacientes zurdos.

\section{Efecto del grado Fisher}

El grado Fisher se muestra predictivo en la variable movimientos alternos de las manos, revelando que manteniéndose constantes el resto de variables, un paciente con grado Fisher II tiene mejor puntuación que la condición control que el grado Fisher I. El grado Fisher también resultó predictivo en las variables detección visual y resta 3 . Si se mantienen constantes el resto de variables, en ambos casos el grado Fisher IV se asocia con una ejecución peor que la condición de referencia Grado Fisher I.

\section{Efecto de la localización del aneurisma}

La localización del aneurisma resultó predictiva en cinco variables. En la variable dígitos hacia atrás, los resultados mostra- ron que, manteniendo constantes el resto de variables, los pacientes con el aneurisma localizado en la arteria comunicante posterior presentan mejores puntuaciones que los pacientes con aneurismas en la arteria comunicante anterior. En las variables copia de la figura semicompleja, recuerdo libre de la figura semicompleja y semejanzas, y si se mantienen constantes el resto de variables, la localización del aneurisma de la bifurcación de la arteria carótida se relaciona con una mejor puntuación en dichas variables. En cambio, los pacientes con el aneurisma de la bifurcación de la carótida presentan una puntuación menor que los pacientes con aneurisma en arteria comunicante anterior en la variable recuerdo con claves de la información verbal.

\section{Efecto de la modalidad de tratamiento}

La modalidad de tratamiento sólo resultó predictiva en las variables recuerdo libre de la figura semicompleja y recuerdo con claves de la información verbal, aunque mostró tendencia a la significación en las variables memoria verbal (codificación) ( $p=$ $0.061)$, copia de la figura semicompleja ( $p=0.094)$, comprensión $(p=0.094)$ y semejanzas $(p=0.069)$. En todos los casos, y manteniéndose constantes el resto de variables de las ecuaciones, los pacientes intervenidos quirúrgicamente presentan menor puntuación que los pacientes tratados endovascularmente. Sin embargo, a pesar de esta tendencia, el cambio en $\mathrm{R}^{2}$ sólo se produce de manera estadísticamente significativa en las variables recuerdo de la figura semicompleja $\left(\mathrm{F}_{1,89}=4.155, p=0.044\right)$ y recuerdo con claves de la información verbal $\left(\mathrm{F}_{1,89}=4.765 ; p=0.032\right)$. Por lo tanto, es en estas dos variables donde la modalidad de tratamiento resulta un buen predictor a pesar del efecto que puedan tener las demás variables independientes incluidas en el modelo.

\section{Discusión}

Nuestro primer objetivo fue describir el estado neuropsicológico de los pacientes con aneurismas cerebrales. Encontramos que la función más frecuentemente afectada en los pacientes tratados quirúrgicamente es la comprensión, mientras que la tarea dígitos es la que presenta menor frecuencia de deterioro neuropsicológico. En el grupo de pacientes tratados con embolización, la tarea memoria verbal (codificación) es la que se muestra deteriorada en el mayor número de pacientes (tabla 3). El porcentaje de pacientes con todas las variables intactas alcanza el $35.7 \%$ en el grupo tratado con cirugía y el $43.2 \%$ en el grupo tratado con embolización. Estos datos se asemejan a los publicados desde hace años en la literatura ${ }^{2,18,20,23}$.

En segundo lugar, nos planteamos determinar en nuestra serie, si existen diferencias en el rendimiento neuropsicológico entre los grupos de pacientes tratados con cirugía o con embolización. Al comparar las medias de los dos grupos, mediante análisis T de Student, encontramos que el grupo de tratamiento endovascular presenta una ejecución mejor que el grupo tratado quirúrgicamente 
en las variables copia de la figura semicompleja, recuerdo libre de la figura semicompleja, recuerdo libre de la información verbal, recuerdo con claves de la información verbal y semejanzas. Sin embargo, hemos de considerar que ambos grupos diferían en la localización del aneurisma, así come en la dominancia manual y en la distribución del grado Fisher, aunque estas dos últimas variables no alcanzaron la significación estadística. Dado que estas variables podrían explicar las diferencias encontradas en los análisis T de Student, realizamos otros análisis que nos permitieran controlar el efecto de dichas variables. De este modo, se realizó un análisis de regresión lineal por variable neuropsicológica, incluyéndose como predictores esas variables y la modalidad de tratamiento. Mediante estos análisis encontramos que la intervención quirúrgica mantiene su efecto en dos variables, recuerdo libre de la figura semicompleja y recuerdo con claves de la información verbal.

Por lo tanto, en nuestra serie, la modalidad de tratamiento afecta a dos variables de memoria, que presentan un peor pronóstico en el grupo de tratamiento quirúrgico. La memoria es una función que puede verse afectada principalmente tras la afectación de los lóbulos temporales y frontales. Los daños en los lóbulos temporales se asocian con más frecuencia a la presencia de deterioro en la función de codificación. Una de las variables afectadas, recuerdo con claves de la información verbal, puede estar indicando la presencia de alteraciones en la codificación de la información verbal. En consecuencia, es posible que la intervención quirúrgica produzca más daños en los lóbulos temporales que el tratamiento endovascular. El efecto de la intervención podría residir, como proponen Hadjivassiliou y colaboradores ${ }^{9}$, en el clipaje temporal que se emplea frecuentemente en la cirugía. De hecho, la oclusión temporal de los vasos sanguíneos se ha asociado con mayores deterioros neuropsicológicos y de la calidad de vida en pacientes intervenidos quirúrgicamente ${ }^{29}$.

No obstante, y a pesar del efecto de la modalidad de tratamiento, hemos de señalar que el porcentaje de varianza explicado es muy bajo, y que el modelo no presenta, por tanto, un buen ajuste. De este modo, deben de existir otros factores que expliquen mejor el deterioro observado tanto en el grupo de tratamiento quirúrgico como endovascular. Consideramos, al igual que las propuestas de Hütter et al. ${ }^{13,14}$ y Hadjivassiliou et al. ${ }^{9}$, que es la propia hemorragia la que produce daños locales y difusos, que podrían explicar mejor la ejecución en las pruebas neuropsicológicas.

Aparte del objetivo de este estudio, los resultados han mostrado el efecto de otra serie de variables que creemos se han de considerar. En primer lugar, se ha hallado que los pacientes zurdos presentan una ejecución peor en las tareas de memoria a largo plazo (recuerdo libre de la figura semicompleja, recuerdo libre y recuerdo con claves de informa- ción verbal). Estos datos indican que la función mnésica de los pacientes zurdos es más sensible al daño cerebral producido por la propia hemorragia subaracnoidea. Esto podría deberse a que las personas con dominancia corporal izquierda tienen más frecuentemente una distribución bilateral de las funciones cerebrales que las personas diestras, generalmente, se encuentran más lateralizadas. De este modo, al igual que ocurre en la lateralización del lenguaje, podría ocurrir que nuestros pacientes zurdos, en lugar de presentar el patrón más común de organización cerebral para la memoria (lóbulo temporal izquierdo dominante para la memoria verbal y lóbulo temporal derecho dominante para la memoria visual), presenten una organización bilateralizada en la que ambos hemisferios participen por igual en el procesamiento mnésico tanto de material visual como verbal. Así, al provocar la hemorragia subaracnoidea daños uni o bilaterales en los lóbulos temporales podría verse afectada más gravemente la función mnésica en los pacientes zurdos, ya que requiere del funcionamiento de ambos hemisferios para un correcto procesamiento.

Otro de los efectos que hemos encontrados es la influencia de la extensión de la hemorragia expresado por la escala Fisher. Los resultados muestran que una mayor $\mathrm{s}$ gravedad en el Fisher (grado IV) se relaciona con menores puntuaciones en las tareas atencionales de detección visual y resta 3 . Sin embargo, los resultados han mostrado que en la variable movimientos alternos de las manos, un grado Fisher más grave (grado II) presenta una ejecución mejor que un grado Fisher I. Consideramos que este efecto podría deberse a diferencias en la distribución de otras variables, como por ejemplo, el hecho de que en nuestra serie haya más pacientes con Fisher II entre los tratados con embolización. De igual modo, la distribución diferencial de otras variables clínicas, periquirúrgicas o sociodemográficas podrían explicar estos resultados.

Por último, en relación a la localización del aneurisma comprobamos que ésta puede influir en la ejecución neuropsicológica de los pacientes. En general, los resultados han mostrado que los aneurismas en la arteria comunicante anterior se relacionan con una peor ejecución en diversas tareas, (copia de la figura semicompleja, recuerdo libre de la figura semicompleja y semejanzas), en comparación con los pacientes con aneurismas de la bifurcación carotídea. Por lo tanto, parece que los daños asociados al tratamiento del aneurisma de la arteria comunicante anterior afectan más a la praxia visoconstructiva, a la memoria visual y a la función ejecutiva. También hemos encontrado que los pacientes con el aneurisma de la comunicante anterior presentan una ejecución peor en la tarea atencional dígitos hacia atrás, en comparación a los pacientes con el aneurisma de la arteria comunicante posterior. Esto podría deberse a los mayores daños en el tejido, que puede provocar la interrupción de aporte sanguíneo a través de los 
vasos perforantes. No obstante, la ejecución en la variable recuerdo con claves de la información verbal es peor en el grupo de pacientes con aneurisma de la bifurcación de la carótida que en el grupo de la comunicante anterior. Estos últimos datos resultan contradictorios con aquéllos que muestran un mayor deterioro neuropsicológico en los pacientes con aneurismas en la arteria comunicante anterior. De nuevo, consideramos que estos resultados podrían deberse al efecto de la distribución desigual en nuestra serie de otras variables entre los pacientes con el aneurisma de la arteria comunicante anterior y en la bifurcación de la carótida.

Los diferentes hallazgos encontrados se han de considerar con cautela, debido a las limitaciones que presenta este trabajo. En primer lugar, este es un trabajo retrospectivo y se ha perdido la información de numerosos pacientes que no pudieron participar. Es posible que algunos de los pacientes que no participaron en el estudio no lo hicieran por encontrarse más limitados, al padecer secuelas más graves. Por lo tanto, no podemos asegurar que estos datos sean aplicables a toda la población de pacientes. Metodológicamente, hemos de considerar que hubiera sido adecuado asignar aleatoriamente los pacientes a las modalidades de tratamiento quirúrgico o endovascular.

En cambio, este procedimiento hubiera llevado a la exclusión del estudio de aquellos pacientes que no podían ser tratados al azar, ya que por la características del aneurisma se hacía necesaria una sola modalidad de tratamiento. Así, en nuestro trabajo, si bien la adecuación metodológica es menor, se describe un rango más amplio de población con hemorragia subaracnoidea, al incluirse aquellos pacientes que necesariamente habían de recibir tratamiento quirúrgico. Además, para controlar las variables en las que ambos grupos no eran homogéneos, se han aplicado procedimientos estadísticos para controlar el efecto de las variables clínicas y demográficas en las que diferían los grupos. También, hemos de considerar las limitaciones de la batería empleada, ya que es una batería para realizar un reconocimiento rápido y amplio de la función cognitiva. Hemos encontrado que algunas tareas presentan efecto techo y por lo tanto, podrían no ser lo suficientemente sensibles para detectar diferencias entre los pacientes. Respecto al tipo de pruebas empleadas, hemos de tener en cuenta las limitaciones de las tareas de memoria visual basadas en la copia previa de una figura. En el caso de que existan deterioros en la praxia visoconstructiva responsable de la copia, la ejecución en memoria posterior se explicaría por dichas alteraciones y no por un déficit en memoria propiamente dicho. Este tipo de problemas podrían salvarse empleando diversas pruebas para evaluar diferentes funciones, incrementándose así la sensibilidad de la evaluación.

\section{Conclusión}

En conclusión, hemos encontrado que la modalidad de tratamiento afecta neuropsicológicamente a los pacientes de forma estadísticamente significativa en las funciones de memoria visual y de recuerdo verbal con claves, que se encuentran más afectadas en el grupo que recibió tratamiento quirúrgico que en el tratado por vía endovascular. Esto podría deberse a un mayor daño en los lóbulos temporales que afectara al proceso de codificación de la información. No obstante, y dado que el porcentaje de varianza explicado por la modalidad de tratamiento es muy bajo, creemos que en la explicación del deterioro neuropsicológico de estos pacientes parece tener más peso el propio efecto de la hemorragia subaracnoidea sobre el tejido que la modalidad de tratamiento.

\section{Bibliografía}

1. Berry, E., Jones, R.A., West, C.G., Brown, J.D.: Outcome of subarachnoid haemorrhage. An analysis of surgical variables, cognitive and emotional sequelae related to SPECT scanning. Br J Neurosurg 1997; 11: 378-87.

2. Bjeljac, M., Keller, E., Regard, M., Yonekawa, Y.: Neurological and neuropsychological outcome after SAH. Acta Neuroehir Suppl (Wien) 2002; 82: 835.

3. Chan, A., Ho, S., Poon, W.S.: Neuropsychological sequelae of patients treated with microsurgical clipping or endovascular embolization for anterior communicating artery aneurysm. Eur Neurol 2002; 47: 37-44.

4. De Renzi, E., Vignolo, L.A.: The Token Test: A sensitive test to detect disturbances in aphasic. Brain 1962; 85: 665678.

5. De Santis, A., Laiacona, M., Barbarotto, R., et al.: Neuropsychological outcome of patients operated upon for an intracranial aneurysm: analysis of general prognostic factors and of the effects of the location of the aneurysm. J Neurol Neurosurg Psychiatry 1989; 52: 1135-1148.

6. De Santis, A., Laiacona, M., Barbarotto, R., De Divitiis, O., Migliore, M., Capitani, E.: Neuropsychological outcome of operated cerebral aneurysms: prognostic factors on 148 patients. Acta Neurol Scand 1998; 97: 393-397.

7. Fontanella, M., Perozzo, P., Ursone, R., Garbossa, D., Bergui, M.: Neuroppychological assessment alter microsurgical clipping of endovascular treatment for anterior communicating artery aneurysm. Acta Neurochir (Wien) 2003; 145: 867-872.

8. Gluglielmi, G., Vinuela, F., Sepetka, I., Macellari, V.: Electrothrombosis of saccular aneurysms via endovascular approach. J Neurosurg $1991 ; 75: 1-7$.

9. Hadjivassiliou, M., Tooth, C.L., Romanowski, C.A., et al.: Aneurysmal SAH: cognitive outcome and structural damage after clipping or coiling. Neurology 2001; 56: 1672-1677. 
10. Hernesniemi, S., Vapalhati, M., Niskanen, M., et al.: One year outcome in early aneurysin surgery: a 14 years experience. Acta Neurochir (Wien) 1993; 122: 1-10.

11. Hillis, A.E., Anderson, N., Sampath, P., Rigamonti, D.: Cognitive impairments after surgical repair of ruptured and unruptured aneurysms. J Neurol Neurosurg Psychiatry 2000; 69: 608-615.

12. Hutter, B.O., Gilsbach, J.M.: Which neuropsychological deficits are hidden behind a good outcome (Glasgow $=$ I) after aneurysmal subarachnoid hemorrhage? Neurosurgery 1993; 33: 999-1005.

13. Hutter, B.O., Kreitschmann-Andenmahr, I., Gilsbach, J.M.: Health-related quality of life after aneurysmal subarachnoid hemorrhage: impacts of bleeding severity, computerized tomography findings, surgery, vasospasm, and neurological grade. J Neurosurg 2001; 94: 241-251.

14. Hutter, B.O., Kreitschmann-Andermahr, I., Mayfrank, L., Rohde, V., Spetzger, U., Gilsbach, J.M: Functional outcome after aneurysmal subarachnoid hemorrhage. Acta Neurochir (Wien) Suppl 1999; 72: 157-174.

15. International Subarachnoid Aneurysm Trial (ISAT), collaborative group: International Subarachnoid Aneurysm Trial (ISAT) of neurosurgical clipping versus endovascular coiling in 2143 patients with ruptured intracranial aneurysms: randomized trial. The Lancet 2002; 360: 1267-1274.

16. Kassel, N.F., Torner, J.C., Holey, C.E., Jone, J.A., Adams, H.P., Konpable, P.L.: The international cooperative study on the timing of aneurysmal surgery. Part 1: Overall management result. J Neurosurg 1990; 73: 18-36.

17. Koivisto, T., Vanninen, R., Hurskainen, H., Tapani, S., Hernesniemi, J., Vapalhati, M.: Outcomes of early endovascular versus surgical treatment of ruptured cerebral aneurysms. Stroke 2000; 31: 2369-2377.

18. Kreiter, K.T., Copeland, D., Bernardini, G.L., et al.: Predictors of Cognitive dysfunction after subarachnoid hemorrhage. Stroke 2002; 33: 200-208.

19. Larsson, C., Furssell, A., Ronnberg, J., Lindberg, M., Nilsson, L.G., Fodstad, H.: Subarachnoid blood on CT and memory disfunctions in aneurysmal subarachnoid hemoriliage. Acta Neurol Scand 1994; 90: 331-336.

20. Ljunggren, B., Sonesson, B., Saveland, H., Brandt, L.: Cognitive impairment and adjustment in patients without neurological deficits after aneurysmal SAH and early operation. J Neurosurg 1985; 62: 673-679.

21. Lot, G., Houdart J., Cophignon, J., Casaco, A., George, B.: Combined management of intracraneal aneurysm by surgical and endovascular treatment. Modalities and results from a serie of 395 cases. Acta Neurochir (Wien) 1999; 141: 557562 .

22. Hillis, A.E., Anderson, N., Sampath P, Rigamonti D.: Cognitive impairments after surgical repair of ruptured and unruptured aneurysms. J Neurol Neurosurg Psychiatry 2000, 69: 608-615.
23. Ogden, J.A., Mee, E.W., Henning, M.: A prospective study of impairment of cognition and meinory and recovery after subarachnoid hemorrhage. Neurosurgery 1993; 33: 572586.

24. Ostrosky-Solís, F., Ardila, A., Rosselli, M.: NEUROPSI: Evaluación Neuropsicológica Breve en Español. Manual, Instructivo y Protocolo de Aplicación [NEUROPSI: A brief neuropsychological evaluation in Spanish. Manual, instructions, and application protocol]. D.F. México; Bayer de México, 1997.

25. Ostrosky-Solís, F., Ardila, A., Rosselli, M.: NEUROPSI: A brief neuropsychological test battery in Spanish with norms by age and educational level. J Int Neuropsychol Soc 1999; 5: 413-433.

26. Rey, A .: Test de copie et de reproduction de mémorie de figures géométriques complexes. París ; Edition du Centre de Psychologie Appliquée, 1959.

27. Ronmer, B., Sonesson, B., Ljunggren B., Brandt., L., Sáveland, H., Holtas, S.: Late magnetic resonance imaging related to neurobehavioral functioning of ASH. Neurosurgery 1989; 25: 390-397.

28. Satzger, W., Niedermeier, N., Schonberger, J., Engel, R.R., Beck, O.J.: Timing of operation for ruptured cerebral aneurysm and long-term recovery of cognitive functions. Acta Neurochir (Wien) 1995; 136: 168-174.

29. Tidswell, P., Dias, P.S., Sagar, H.J., Mayes, A.R., Battersby, R.D.: Cognitive outcome after aneurysm rupture: relationship to aneurysm site and perioperative complications. Neurology 1995; 45: 875-882.

30. Weschsler, D. WAIS-R manual. New York; The Psychological Corporation, 1981.

\section{Agradecimientos}

Agradecemos a María del Mar Fernández de Águila su asesoramiento y guía en la realización de los análisis estadísticos de este trabajo.

Orozco-Giménez, C.; Katati, M.J.; Vilar, R.; Meersmans, M.; Pérez-García, M.; Martín, J.M.; Alcázar, P.; Guerrero, F.; Escamillo, F.; Mínguez, A.; Olivares, G.; Saura, E.; Jorques, A.; Arjona, V.: Alteraciones neuropsicológicas en pacientes con aneurismas cerebrales: tratamiento quirúrgico versus tratamiento endovascular. Neurocirugía 2006; 17: 34-45.

Correspondencia postal: Majed J. Katati. Servicio de Neurocirugía. Hospital Universitario Virgen de las Nieves. Ctra Jaén s/n. 18012, Granada (España).

Este trabajo ha sido financiado con las ayudas a proyectos de investigación otorgadas por la Fundación Virgen de las Nieves (Hospital Universitario Virgen de las Nieves). 
Comentario al trabajo: Alteraciones neuropsicológicas en pacientes con aneurismas cerebrales de Orozco Giménez y cols.

Orozco y colaboradores se aproximan de nuevo a la cuestión de las diferencias entre el tratamiento quirúrgico y endovascular de los aneurismas cerebrales, pero esta vez desde el enfoque de las consecuencias neuropsicológicas en estos pacientes, aspecto al que probablemente no se presta toda la atención debida en nuestro entorno.

El trabajo parte de unas limitaciones que los autores detectan adecuadamente y soslayan en lo posible con una metodología apropiada. La discusión analiza de forma minuciosa los resultados obtenidos, aunque alguna de las argumentaciones empleadas para explicar la aparente inferioridad de los pacientes zurdos en la función mnésica tras la hemorragia, o la benevolencia de grados más graves de sangrado en la escala de Fisher en alguna de las funciones cognitivas, parecen poco sólidas.

En las conclusiones, se apunta una superioridad del tratamiento endovascular en dos de los catorce aspectos neuropsicológicos explorados. Esta afirmación debe tomarse con cautela, como los propios autores señalan, tanto por la naturaleza de los datos analizados, como por el bajo porcentaje explicativo del modelo propuesto. En cualquier caso, hay que felicitar el esfuerzo realizado que debería impulsarnos a profundizar desde este punto de vista en la candente cuestión de las dos alternativas terapéuticas.

J. Ibáñez

Barcelona
Comentario al trabajo: Alteraciones neuropsicológicas en pacientes con aneurismas cerebrales de Orozco Giménez y cols.

Este es un interesante trabajo en el que se pone de manifiesto la existencia de importantes trastornos neuropsicológicos en la mayoría de los enfermos (mayor del $60 \%$ en este trabajo) que han presentado buena evolución según escalas tradicionalmente usadas en la descripción de la evolución de procesos neuroquirúrgicos, como la escala de evolución de Glasgow. Dado su carácter retrospectivo, el ser una muestra seleccionada, y la ausencia de un adecuado balance entre los grupos de tratamiento quirúrgico y embolizador, dado que no existe una aleatoriedad en el tratamiento, quizás la mayor crítica que se puede hacer al trabajo es su excesivo interés en establecer con sus hallazgos una diferencia entre ambos tratamientos. Es, sin embargo, interesante observar cómo en ambos tratamientos la frecuencia de afectación neuropsicológica es similar, factor que apoya el hecho de que es el sangrado inicial el que determina esta afectación.

Por ello, animaría a los autores a investigar, de forma global y no en la búsqueda de diferencias entre tratamientos, los factores que influyen en el desarrollo de déficits neuropsicológicos. Sería interesante, y es esperable que los autores habrán explorado este factor, la relación entre la presencia y gravedad de la afectación neuropsicológica y la gravedad clínica del sangrado inicial, expresada por el nivel de conciencia del enfermo. Aunque la ausencia de diferencias entre tratamientos puede indicar la relación entre el sangrado y su gravedad, sería fácil para los autores comprobar, con datos, esta posibilidad. Los autores podrían establecer mediante el mismo análisis, cuáles son los factores que han afectado a la evolución neuropsicológica en el conjunto de su muestra, incluyendo todos los factores, sobre todo aquellos más relacionados con el pronóstico global de estos enfermos como son la edad y el nivel de conciencia al ingreso. Esta información sí sería importante y probablemente, de esta forma, la varianza explicada por estos modelos aumente y supere el escaso $20 \%$ que muestran los mejores de ellos.

De todas formas, es de agradecer el esfuerzo realizado por los autores en el seguimiento exhaustivo de sus enfermos. 